\title{
CAD based shape optimization for gas turbine component design
}

\author{
Djordje Brujic *†, Mihailo Ristic $\uparrow$, \\ Massimiliano Mattone $\ddagger$, Paolo Maggiore $\ddagger$ \\ and Gian Paolo De Poli $\S$
}

† Imperial College London, UK

+ Politecnico di Torino, Italy

$\S$ Avio SpA, Italy

Email: d.brujic@imperial.ac.uk

Phone: $+44(0) 2075947175$

Fax: +44 (0) 8700517575

\begin{abstract}
In order to improve product characteristics, engineering design makes increasing use of Robust Design and Multidisciplinary Design Optimisation. Common to both methodologies is the need to vary the object's shape and to assess the resulting change in performance, both executed within an automatic loop. This shape change can be realised by modifying the parameter values of a suitably parameterised Computer Aided Design (CAD) model. This paper presents the adopted methodology and the achieved results when performing optimisation of a gas turbine disk. Our approach to hierarchical modelling employing design tables is presented, with methods to ensure satisfactory geometry variation by commercial CAD systems. The conducted studies included stochastic and probabilistic design optimisation. To solve the multi-objective optimisation problem, a Pareto optimum criterion was used. The results demonstrate that CAD centric approach enables significant progress towards automating the entire process while achieving a higher quality product with the reduced susceptibility to manufacturing imperfections.
\end{abstract}

\author{
Keywords \\ design optimisation, robust design, parametric CAD modelling, gas turbine
}

\section{Introduction}

Engineering design makes increasing use of methodologies such as Multidisciplinary Design Optimisation (MDO) and Robust Design (RD). In this paper their application in situations where the geometry of a component is to be optimised in order to achieve certain goals is considered. Geometry optimisation requires variation of the object shape and assessment of the resulting change in the performance (Haslinger and Makinen 2003). This is common to both MDO and RD methodologies.

MDO is concerned with achieving a design that simultaneously satisfies the requirements and optimises the performance in different disciplines. In aerospace engineering this may involve optimisation of parameters by considering the combined structural, thermal and aerodynamic performance.

Robust design on the other hand is fundamentally concerned with minimizing the effect of uncertainty or variation in the design parameters without eliminating the source of that uncertainty or variation (Kalsi et al 2001). In other words, a robust design is 'less sensitive' to variations in uncontrollable design parameters than the traditional optimal design. Robust design has found many successful applications in engineering and is continually being expanded to different design 
phases. Although robust design has been traditionally applied in manufacturing there has been research recently into applying these techniques to make the design conceptually robust. The important roles of modelling and calculation of robustness in a multidisciplinary design environment is discussed in (Marczyk 2000).

Realisation of MDO and RD processes inevitably requires close integration of functions such as geometric design, engineering analysis (e.g. finite element) and optimisation algorithms, (Bennet et al 1998). Such functions are today extensively supported by commercial software packages which may be used in combination to achieve maximum benefits. Modern CAD systems (e.g. Catia, Pro/E, Unigraphics) are used as the central tool for creating and maintaining product definition throughout its lifecycle. They provide a rich set of tools for creation and management of geometry, ranging from parts to complex assemblies, databases of material properties and, increasingly, encapsulation of specialist design methods (e.g. UG Knowledge Fusion). Analysis packages (e.g. MSc Software, Ansys) include extensive pre- and post-processing functions together with solvers dedicated to specific disciplines. Optimisation methods may involve Newton or quasi-Newton type algorithms, while evolutionary and probabilistic methods are increasingly used. Such methods may be implemented using bespoke code, while there is also an increasing number of software packages offering such functionality (e.g. modeFrontier, MSC/Robust Design, iSIGHT).

The optimisation process is characterized by significant human involvement needed to develop the CAD model, to generate the analysis models, to execute the analysis code and finally to examine the output and make decisions. Since the analysis task may require a considerable computational time, automation of the overall procedure is the key to realising higher design productivity. Thus the design practitioners are increasingly interested in methods for integration of such software into an automatic optimisation loop in order to perform difficult optimisation tasks involving multiple design objectives and constraints. An important practical issue is that many of the relevant software tools, especially CAD, are primarily intended for standalone interactive use and their integration into an automatic loop demands special attention.

This paper presents results of the research that has been conducted under the auspices of the EU Framework 6 project VIVACE (Value Improvement through a Virtual Aeronautical Collaborative Enterprise) - a consortium of about 70 European aerospace manufacturers and academic institutions. Among the many aspect of this large project, the central theme has been the provision of methods and tools to enable close integration between various disciplines and tools involved in modern aeroengine design aimed at meeting the overall design targets such as thrust, weight and service life. These include thermal cycle analysis, aerodynamic performance, vibration analysis of the whole engine, coupled with structural, thermal and fatigue life analysis of individual components. Robustness of the final design in the context of multidisciplinary design optimisation is an overriding requirement.

The design case considered here involves shape optimisation of a high pressure gas-turbine disc of an aircraft engine (Fig. 1). The high pressure disk is treated as a generic example of a large class of complex objects that are represented as solids of revolution and/or extrusions. In an aero engine such components do not directly affect the gas flow but are critical for the overall weight, fatigue life and vibration characteristics. Disk design involves two main aspects that are addressed independently. The first is the design of the disc shape, aimed at minimising the weight while maximising the life by maintaining the stresses in critical areas within the prescribed limits. The second is the optimisation of the disk slot and blade root, which provides the interface between the two components. In both cases the overall objective is to achieve an optimal design while ensuring that the design is robust in the presence of uncertainties.

\section{Geometric modelling for shape optimisation}

There are, basically two approaches to CAD and CAE integration (Lee 2005):

- CAE-centric approach

- CAD-centric approach

In the CAE-centric process, engineering analyses are performed initially to define and refine a design concept using idealized analysis models before establishing the CAD model of the product. The design process usually starts with the simplest idealisations of a solid geometry and progresses to more complex ones. CAE geometry typically involves lines or sheets, from which the 3D model 
may be subsequently generated by adding detail and dimensional information. Techniques proposed to carry out dimensional addition and to create solids from abstract models involve sheet thickening, offsetting, and skeleton re-fleshing operations. (Lee, Armstrong, Price and Lamont 2005), but this is not well supported by current systems. CAE geometry cannot be easily used to construct a CAD model, nor other instances of CAE geometry at different levels of abstraction. In practise each such new model needs to be re-created from scratch.

In the CAD-centric approach, the design is captured initially in a CAD system, while the CAE model is derived from that. Since the CAE model usually involves idealisation of the detailed product geometry, many aspects of its creation are supported by the parametric modelling paradigm adopted by the modern CAD systems. For example, simplification of a given solid can often be effectively achieved simply by turning off certain features in the model tree. In other situations however, preparation of the CAE model may involve more complex operations in CAD. For example the CAE model may be represented by a 2D section involving more than one part, which is not available through simple de-featuring and requires explicit geometric operations. Such construction can be performed using available CAD functionality, automated using built-in scripting languages and applied automatically on a family of parts.

Both of these approaches require considerable effort to create and consistently maintain different models for one product, but the CAD-centric approach was considered to offer a number of important advantages. First, it is considered to provide an easier and more natural integration with engineering analysis, especially in situations involving multiple disciplines and complex assemblies. Second, it eliminates any representation related restrictions on allowable geometry changes, which can then be tailored for higher fidelity analysis. Finally, the approach will in the longer term strongly benefit from the continuing advances in CAD functionality, leading to improved productivity.

In this way $\mathrm{CAD}$ becomes the source and repository for all relevant geometric information, including the definition of geometric parameters that are the variables in the optimisation process. The geometric definition can be readily augmented with discipline-specific engineering information such as material properties and boundary conditions. Constraints and influences arising in one discipline and affecting other disciplines are also easier to manage in a complex design scenario.

The drawbacks of this method include the complexity of geometry generation script. Furthermore, it was recognised that existing CAD systems do not robustly support parametric modelling, posing issues for implementation of variational modelling in an automated fashion. Existing practices in parametric modelling, their limitations and technical difficulties are investigated (Shapiro and Vossler 1995). Section 4 of this paper provides details of a pragmatic solution that produced satisfactory results. Raghotama and Shapiro (Raghotama and Shapiro 2002) and Hoffmann and Joan-Arinyo (Hoffmann and Joan-Arinyo, 2002) describe additional limitations of parametric modelling but they are beyond the scope of this paper.

\section{Shape optimisation process}

Shape optimisation can be viewed as part of structural optimisation, a branch of computational mechanics. The methods for structural optimisation are based on selecting a subset of data to be used as parameters, by means of which fine-tuning of the structure is performed until the optimal properties are achieved. Here, the most important aspect is to be able to treat geometry as a variable (Delfour and Zolésio 2001). 


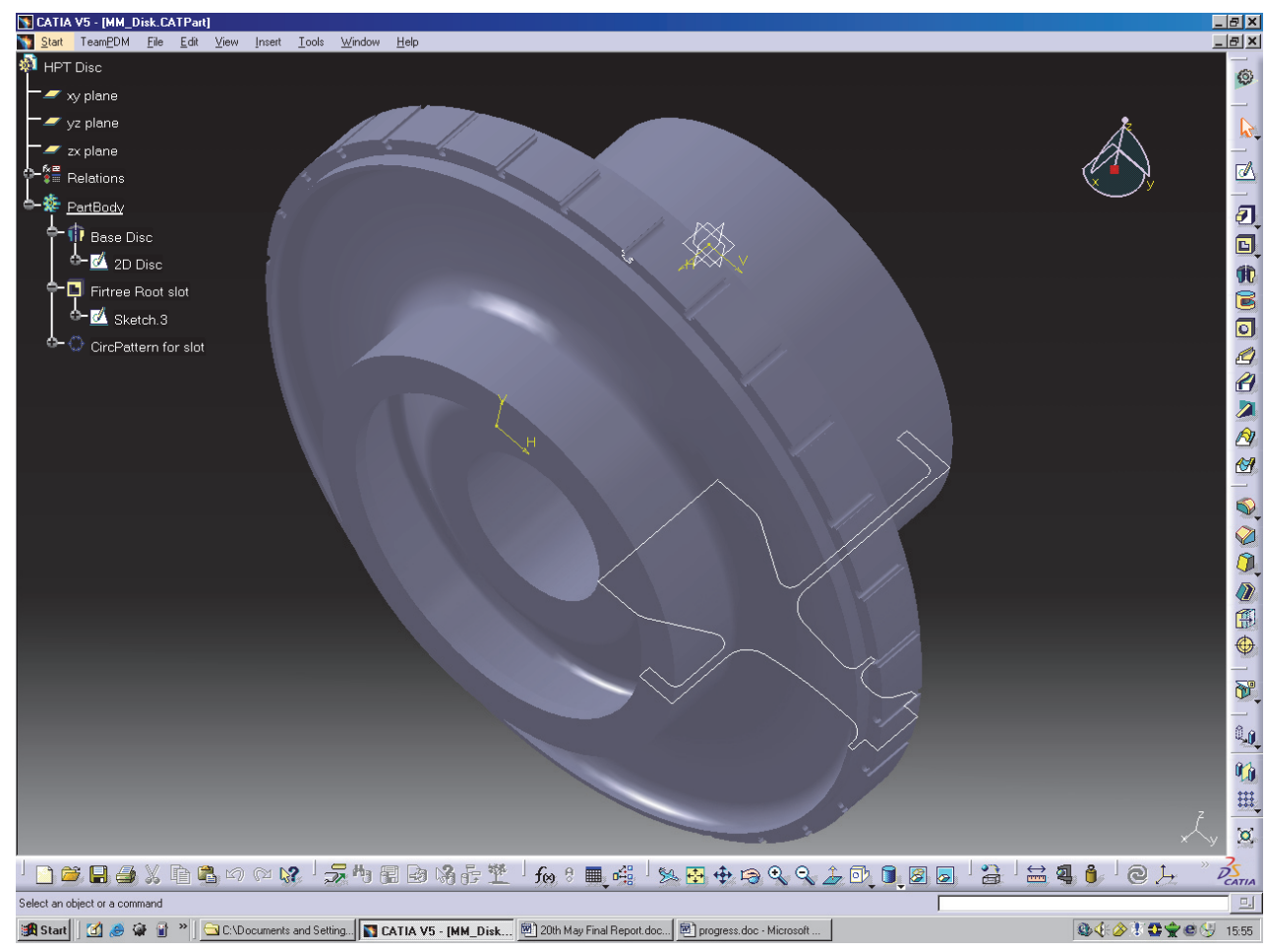

Fig. 1 Gas turbine disc

There are two different ways to implement shape modification within a shape optimisation process. The first one is closely related to the CAE-centric modelling approach (Section 2), where a geometric modelling system initially generates a computational grid from a model. Next, a selection of points on the grid is perturbed and the model re-analysed. This process continues until some desired target or termination condition is reached. Examples of this class of system are MASSOUD (Samareh 2004), DesignTranair (Melvin et al 1999), MDOpt (LeDoux et al 2004) and others (Fenyes et al 2002). This method is limited by the allowable displacement of grid points before the grid becomes inadequate for analysis, inconsistent (e.g., self crossing elements), or violates design constraints (e.g., minimal thickness). The movement of individual points makes shape control difficult to achieve. This type of optimisation is suited for fine tuning of a specific design, but generally it is not suited for large geometry changes. Despite these drawbacks, grid perturbation techniques have proved useful in practice, (Carty and Davis 2004, Nemec et al 2004, Baker et al 2002, Rohl at al 1998).

The second type of shape optimisation moves geometry generation inside the optimisation loop. It generates a new geometry model for each point in the design space, then analyses the design it represents in each of the different disciplines. This is more closely related to the CAD-centric modelling approach and it is better suited in situations when large changes in the geometry occur. We have adopted the second approach, recognising the potential of the parametric modelling paradigm and the fact that it is supported by modern CAD systems. It offers an elegant way to modify the shape while satisfying predefined geometric constraints. Adequately parameterised shape can be controlled by systems external to CAD using the design tables, where each element of the table corresponds to a value of some variable in the design (line length, arc radius, arc angle etc.). These associations, together with the appropriate parameterisation, enable us to achieve above goals.

The steps in the shape optimisation procedure are presented in Fig. 2. The first step is the construction of a parameterised CAD model. Parameterisation of a given shape is not unique, indeed different choices for shape parameters may be better suited for different aspects of design, analysis and manufacture. For shape optimisation, the model must enable automatic generation of a wide range of candidate shapes, where each shape instance must be feasible and adhering to the overall design intent. The design intent is encapsulated in the prescribed relationships between the geometric entities in the model (such as parallelism and tangency) and by the choice and definition of geometric operations used to construct the shape (such as extrusion or filleting) that give rise to the concept of design features. These aspects, together with the relevant parameter values (lengths, radii etc.) represent the parameterised CAD model that is then automatically generated by the CAD system for each new instance of the parameter vector. As today's systems do not allow different parameterisations of one model to coexist, the designer needs to make careful choices 
when devising the CAD model. When the CAD model is the core of the product definition, as adopted by the VIVACE project, then the choice of shape control parameters must primarily adhere to the general principles of Geometric Dimensioning \& Tolerancing (GD\&T).

The second step in Fig. 2 involves selection of the design model, where only subset of the model parameters may be selected for the subsequent optimisation, with the aim to reduce the search space to manageable size.

The third step is a realisation of an automated multidisciplinary optimisation loop. It involves extracting the needed information from the CAD model, modifying the original parameters and executing the relevant simulation code in order to evaluate the performance. The optimisation may be deterministic and/or stochastic. It is important to note that most of the MDO methods in use today require making large changes in the initial shape in order to better characterise the design space and optimise the design according to multiple criteria.

The fourth step involves robustness assessment of the design in relation to the criteria and constraints used in the optimisation. Monte Carlo simulation may be used for this task. It is often the case that an optimised design is shown to be too sensitive to small changes in the design parameters, i.e. small variations in the shape cause large variation in performance. This in turn may pose excessive demands on the allowable tolerances, both dimensional and material properties, with the consequent implications on the cost or even feasibility of manufacture. The final step in the process is the RD optimisation loop. Unlike most MDO methods, RD methods involve small changes of the nominal shape, focussing on the assessment of the effects of manufacturing tolerances and the uncertainty of material properties (Zhang and Wang 1998).

There is also an increasing tendency to combine the two approaches into one process, (Giassi et al 2004).

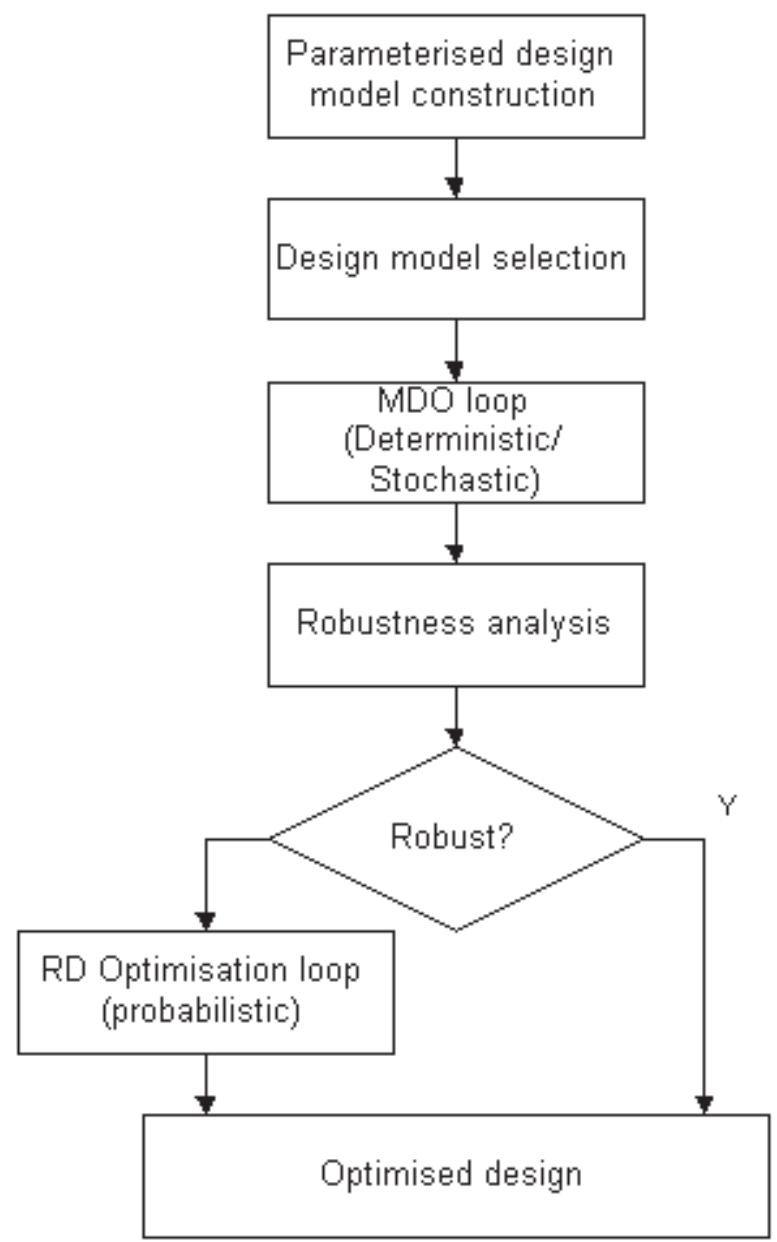

Fig. 2 Typical MDO/RD process flow

The implementation details of relevant optimisation loops are largely determined by the choice of design, analysis and optimisation tools, often involving in-house analysis packages and bespoke programming using Matlab or languages such as $\mathrm{C}++$. For the work presented in this paper integration was realised mainly using Matlab in combination with CAD scripts. In addition, commercial optimisation packages such as iSIGHT/FIPER (www.engineous.com) and 
modeFrontier (www.esteco.com), increasingly offer functionality for integration of different CAD and CAE environments. Suitability of these tools for deployment in a web-based commercial environment was investigated in other parts of the VIVACE project, (Kesseler and Van Houten 2007).

\section{Geometry modeling implementation}

As both MDO and RD are executed in a loop, it is crucial to realise shape change without user interaction. Other considerations include compatibility with collaborative design practices, where multiple, geographically dispersed teams take part in the overall design process. This was efficiently solved through implementation of a hierarchical model structure, where the parametric modelling paradigm allows all parameters to be stored and modified within design tables. This is depicted in Fig. 3 where each box represents a separate file.

At the top level of the model's hierarchy there is an assembly file used as a data collector. In this case it collects the data defining the solid disc and the blade. Three design tables were constructed to control all the design parameters, specifically:

HPT Disc design table - contains 48 numerical parameters of the 2D section defining the disc. Firtree Root design table - contains 20 numerical parameters of which 10 are associated with the slot on the disc and 10 are associated with the corresponding root of the blade. In addition, 11 constants are included in the design table.

Activity design table - contains the commands to switch on/off the features in the disc master model: rotation, extrusion cut and circular pattern. Also, it controls the number of blades by specifying the number of instances for the circular pattern.

An important advantage of the implemented structure is that the shape modifications are introduced at the top level only (within the design tables). Thus, parameter values can be modified either interactively, by the user, or automatically, by a program. The rest of the control structure is updated automatically. The design tables can be implemented as ASCII text files or as Microsoft Excel files.

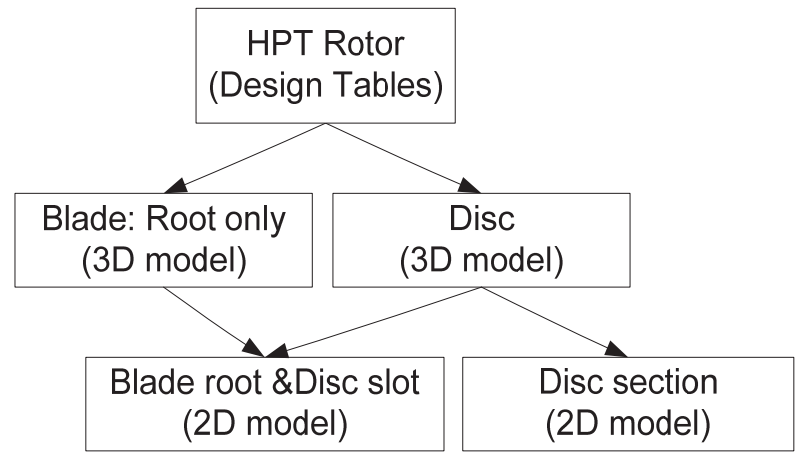

Fig. 3 Model Structure

\section{Parameterisation}

A geometric definition of the problem must be made before starting the optimisation process. The choice of parameters is of paramount importance since it is the equivalent to defining the mathematical model of the optimisation problem. Clearly, it defines the nature and the dimensions of the research space and possible solutions largely depend on it.

Following the modelling structure outlined above, parameterised disc geometry was implemented and tested on two CAD platforms: CATIA V5 and Unigraphics. This highlighted a number of intricate aspects that the designer should consider when defining the model. Fig. 4 and 5 illustrate the full parameterisation of the HPT rotor. Note that for the studies presented here, only the root portion of the blade needed to be modelled in detail, while the rest of the blade was represented by a point mass. 


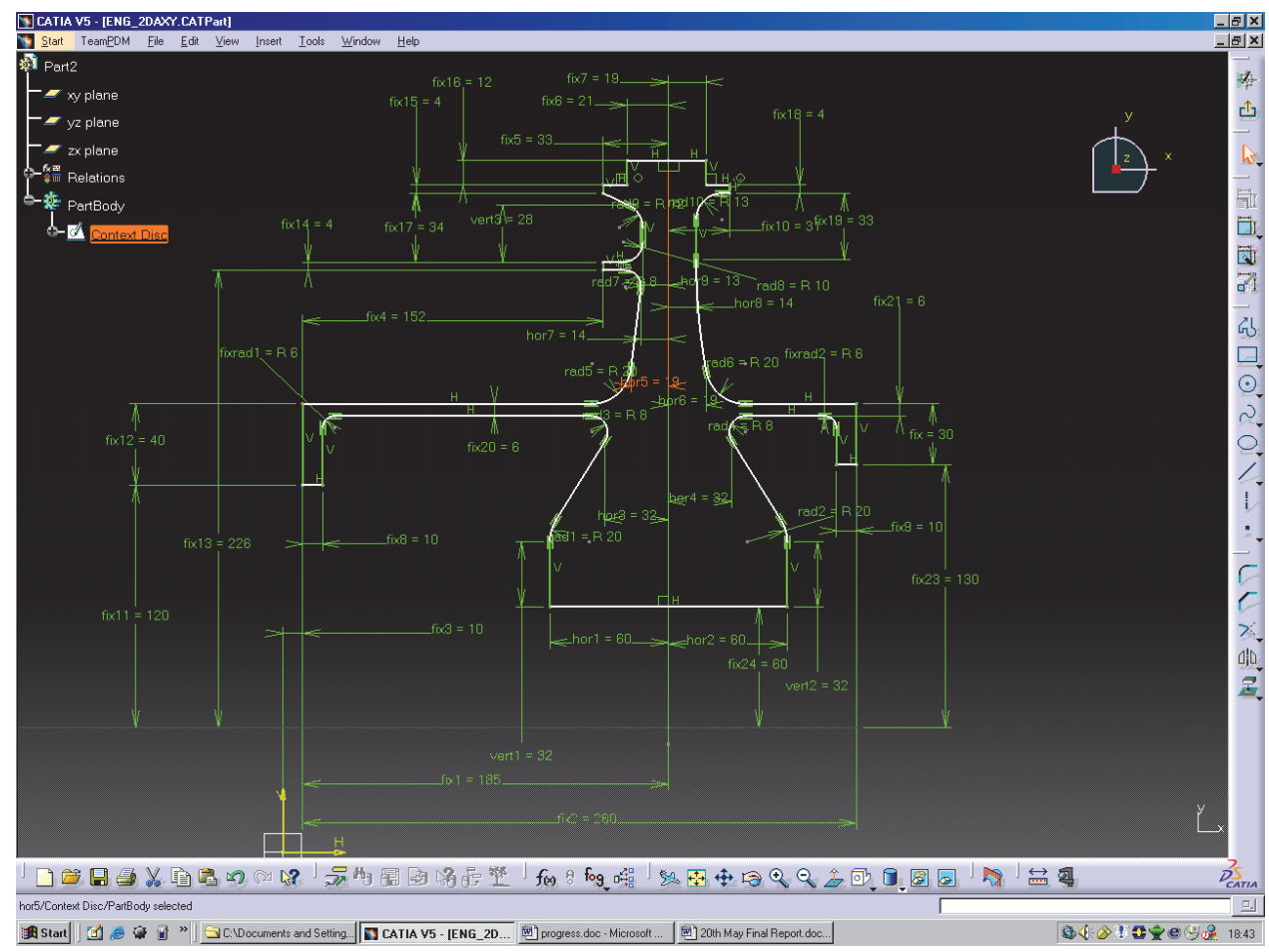

Fig. 4 Parameterized disc

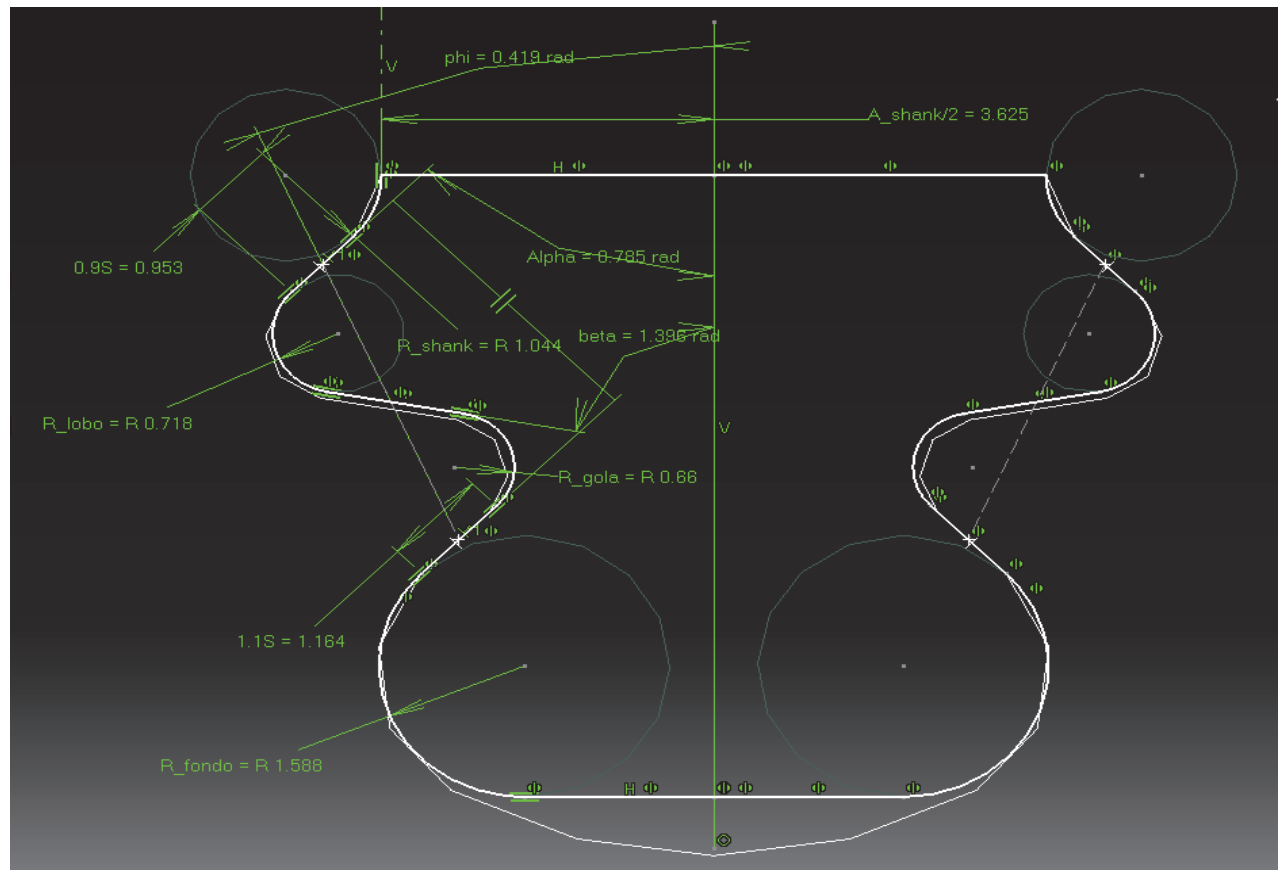

Fig. 5 Parameterised blade root and slot

The optimisation algorithm has to be able to find a relationship between the design variable variations and the evolution of performance values. Thus, a controlled modification of the original disc design was required. This was realised by implementing scripts that enable the complete calculation process to be entirely performed in batch mode.

An important aspect of parameterisation step is the definition of parameter boundaries. At the preliminary design stage these can be used to define a family of parts, while at the optimisation stage they can define the design space within which the optimisation is performed.

For all CAD packages considered, the likelihood of generating infeasible geometry was found to be highly dependent on the choice and size of the parameter subset being varied, as well as the shape in question and parameterisation details. For this reason the permissible parameter boundaries have to be judiciously chosen for each specific optimisation task. In the case of disc optimisation loops considered here, a subset of 8 parameters was varied. 


\section{Model correctness analysis}

The constraints prescribed by the model construction result in a set of simultaneous constraint equations and/or inequalities. These equations are solved for the specific instances of the parameter values by the constraint solver and the geometry of the part is regenerated accordingly within the CAD package whenever a parameter value is modified (Hoffman and Joan-Arinyo 1998). As the constraint equations are typically non-linear, they require the use of iterative methods. With any iterative method, the convergence strongly depends on the value of the initial guess in relation to the solution. If the initial guess is far from the correct solution, the method can converge to a wrong solution, as illustrated by the disc geometry in Fig. 6. Such a case is easily identified through the validation readily available within a CAD package.

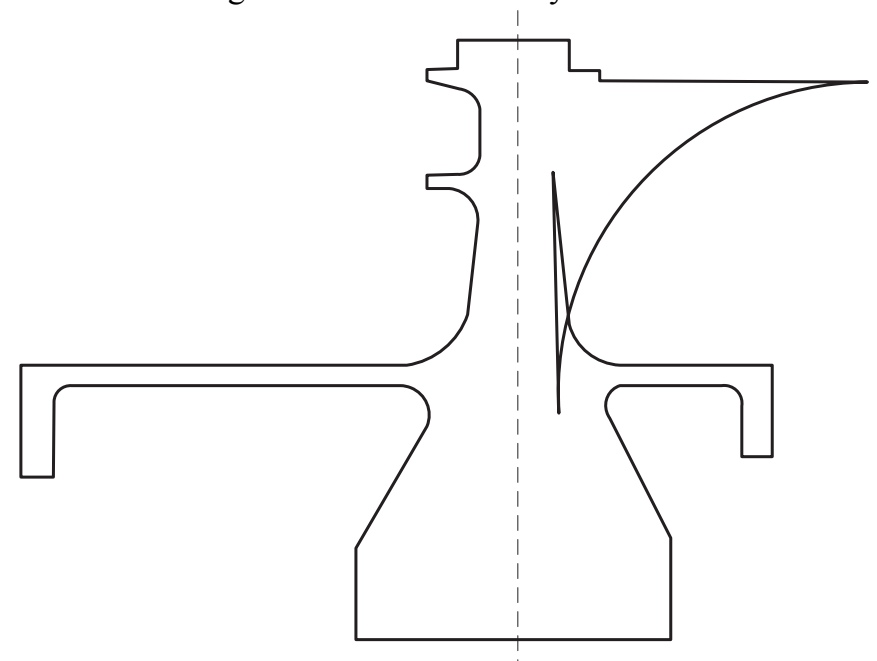

Fig. 6 Example of a non-feasible geometry

On some occasions the method may fail to converge at all, in which case the software simply returns an error message. Bearing this in mind, an important aspect of the parameterisation is to ensure, or at least to have high probability to achieve, the correct shape.

To test the correctness of the design hundreds of simulations involving generation of sets of design parameters within the given range were generated in a random fashion. For each range, 100 random parameter sets were modified around their nominal values using the following formula: $U=U^{*}[(1-x)+2 * x * R n d]$

where $U$ is a design variable, $x$ is a range and Rnd is a random number between 0 and 1 .

Initially, studies were performed by varying all 48 parameters of the disc model. This has shown that the permissible range of parameter variation is less than $2-3 \%$ if high probability of generating feasible geometry is to be achieved.

Subsequent studies involved varying subsets of 8 parameters for the disc and blade root, which were selected as candidates for optimisation and the results are presented in Tables 1 and 2. It can be seen that the limit of allowable range is about $30 \%$. It was also found that smaller jumps between the values are more reliable.

Table 1 Blade root geometry test results

\begin{tabular}{|l|l|l|}
\hline Range (\%) & No. of tests & No. of valid geometries \\
\hline 10 & 100 & 100 \\
\hline 20 & 100 & 100 \\
\hline 30 & 100 & 100 \\
\hline 40 & 100 & 100 \\
\hline 41 & 100 & 98 \\
\hline 42 & 100 & 95 \\
\hline 42.5 & 100 & 92 \\
\hline 45 & 100 & 90 \\
\hline 50 & 100 & 82 \\
\hline 60 & 100 & 67 \\
\hline 70 & 100 & 34 \\
\hline
\end{tabular}

Table 2 HPT Disc geometry test results 


\begin{tabular}{|l|l|l|}
\hline Range (\%) & No. of tests & No. of valid geometries \\
\hline 10 & 100 & 100 \\
\hline 20 & 100 & 100 \\
\hline 30 & 100 & 100 \\
\hline 31 & 100 & 90 \\
\hline 40 & 100 & 74 \\
\hline 50 & 100 & 60 \\
\hline 60 & 100 & 43 \\
\hline 70 & 100 & 27 \\
\hline
\end{tabular}

\section{Disc design and optimisation}

The results of disc optimisation, shown here as an example, were obtained at an early design stage - preliminary shape optimisation.

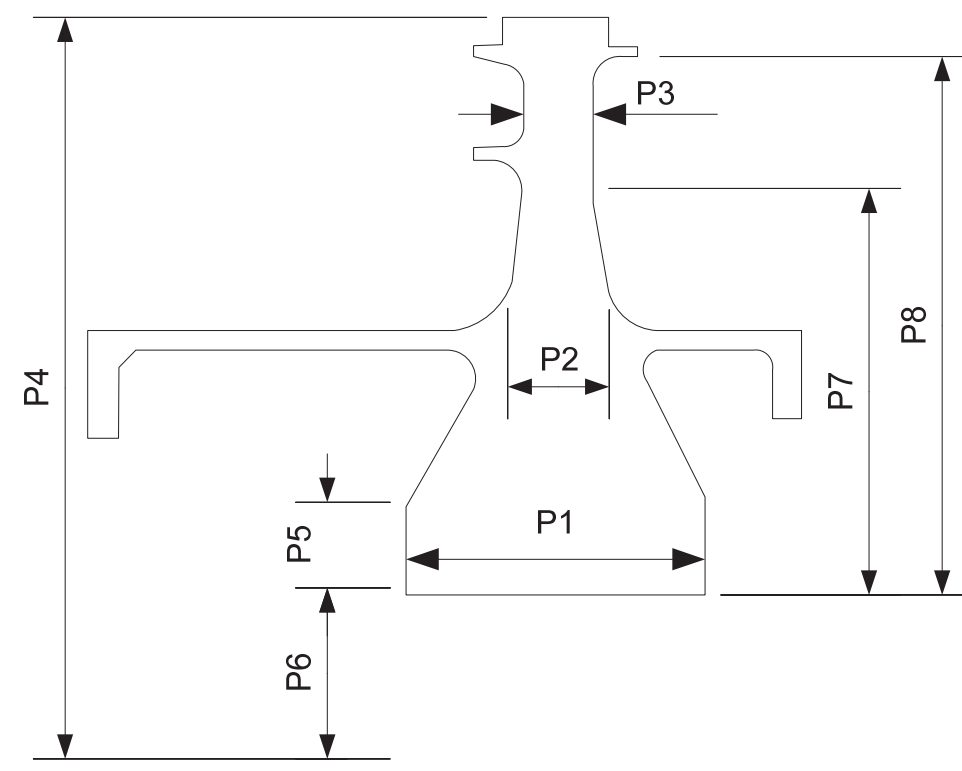

Fig. 7 Parameterisation for preliminary disc design

The objective was to find a minimum-weight shape of the disc, satisfying given constraints that can be defined in terms of maximum stress allowable at a given location, as well as of burst speed and fatigue life. Only the parameters that were considered to be most influential in controlling the overall shape of the disc were optimised, as presented in Fig. 7.

An automated, analysis process was set up to perform the numerical thermo-mechanical calculations. The program was written in MATLAB and performs following actions:

- Launches CATIA and automatically generates the disc shape using an ASCII file containing design variables as input.

- Generates an IGES file needed as the input for the mesh generator.

- Launches the MSC/Patran pre-processor for FE model set up and automatic meshing

- Launches MSC/P-Thermal for the evaluation of the temperature fields

- Launches MSC/Nastran for stress analysis

- Launches MSC/Robust Design to perform optimisation and analysis using Stochastic Design improvement methodology

The communication between different packages is most conveniently realised through files. Some optimisation loops presented in the subsequent sections involve the use of different design and analysis tools, but the overall structure is basically the same.

The design parameter values obtained through the optimisation are presented in Table 3.

Table 3 Disc optimisation results

Parameter Initial Optimised




\begin{tabular}{|lll|}
\hline $\mathrm{p} 1$ & 70 & 64 \\
\hline $\mathrm{p} 2$ & 10 & 12 \\
\hline $\mathrm{p} 3$ & 80 & 84 \\
\hline $\mathrm{p} 4$ & 655 & 650 \\
\hline $\mathrm{p} 5$ & 54 & 50 \\
\hline $\mathrm{p} 6$ & 120 & 144 \\
\hline $\mathrm{p} 7$ & 370 & 355 \\
\hline $\mathrm{p} 8$ & 430 & 424 \\
\hline
\end{tabular}

The minimum weight shape has been calculated imposing that the maximum stress on the disk is smaller than a given value. Starting from this solution, further features and parameters may be considered in order to further control the shape of the disc and to perform further optimisation on new parameters.

\section{Blade root optimisation and robust design}

The blade root design must respect three important constraints:

1. Rupture criteria

2. Geometrical relationship criteria

3. Stress concentration limits in critical areas

The first constraint dictates that the rupture in the blade (critical stress) must occur before the rupture in the disc. Formally, defining $p_{i}$ as the stress reached in section $i$ and $\sigma_{\text {rupture }}$ as the ultimate stress of the blade and disc material (Fig. 8), the dimensionless factor $P_{i}$ is defined as:

$$
P_{i}=p_{i} / \sigma_{\text {rupture }}
$$

The following conditions have to be satisfied with the assigned priority:

$$
\begin{array}{ll}
P_{1}>P_{2} & \text { (mandatory condition) } \\
P_{1}>P_{4} & \text { (mandatory condition) } \\
P_{2}>P_{4} & \text { (desirable condition) }
\end{array}
$$

The second constraint, geometrical relationship criteria, concerns the relative feature sizes of the blade root and the disc. Defining md the smallest sectional area in the disc slot and $\mathrm{mp}$ the smallest sectional area in the blade root (see Fig. 8), the following condition has to be satisfied:

$$
K_{l}<\frac{m_{d}}{m_{d}+m_{p}}<K_{u}
$$

where $K_{b}, K_{u}<1$ are the user specified constants.

The third constraint applies limits on concentrated stress in critical areas. Defining the maximal principal stress component at the critical locations as MPS, the contact pressure between blade root and disc slot as $p r_{-} l$ and $p r_{-} 2$, and the yield stress of the blade and disc material as YTS (Fig. 8), the following constraints are formalised:

$$
\begin{array}{lr}
M P S<Y T S & \text { (mandatory) } \\
p r \_1<K_{y} Y T S & \text { (mandatory) } \\
p r \_2<K_{y} Y T S & \text { (mandatory) }
\end{array}
$$

where $K_{y}<1$ is a constant 

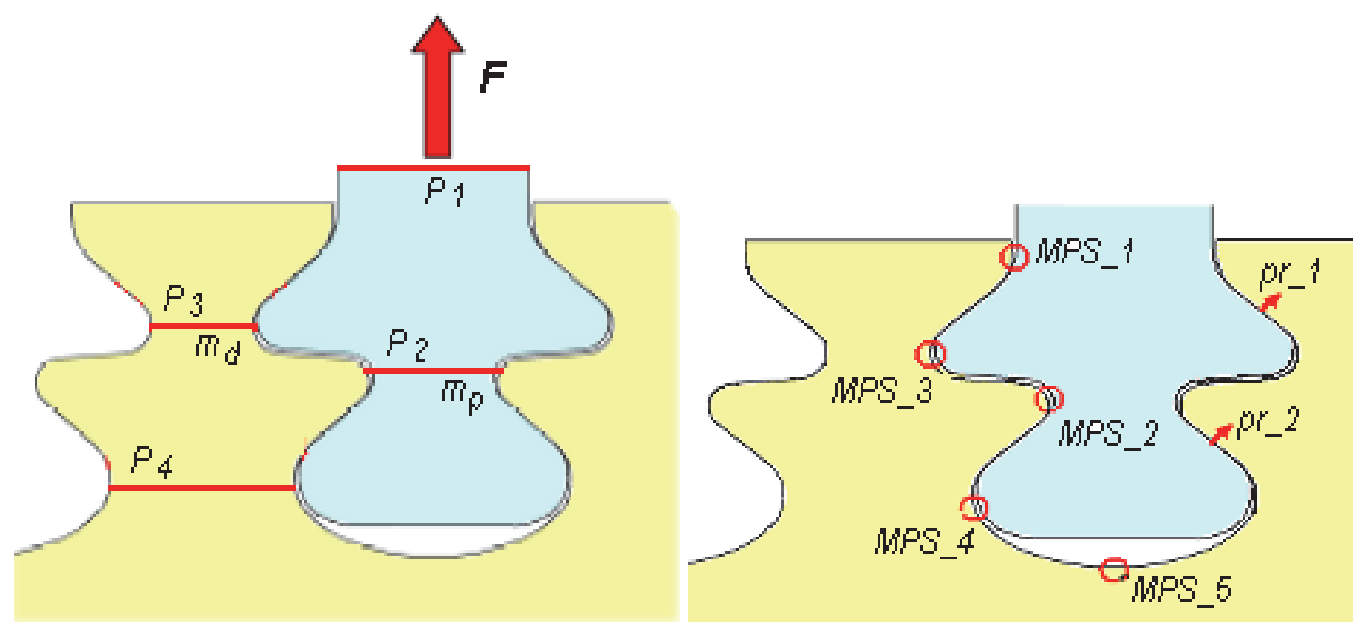

Fig. 8 Blade root and disc slot design

\section{Optimisation}

In order to reduce the design space the optimisation of the blade root was implemented within two separate optimisation processes: meeting the rupture criteria and minimisation of critical stresses. First, the shape of the blade root was optimised with the respect to the rupture criteria. It may be formalised as follows:

Find the set of design variables $X$ that maximises

$P_{1}-P_{2}$

$P_{2}-P_{4}$

\section{Subject to}

$$
K_{l}<\frac{m_{d}}{m_{d}+m_{p}}<K_{u}
$$

Optimisation using the Multi-Objective Genetic Algorithm (MOGA) was implemented within modeFrontier design environment. The Pareto front was subsequently analysed and an optimal solution was identified. The scatter plot of the two objectives with the Pareto front is illustrated in Fig. 9

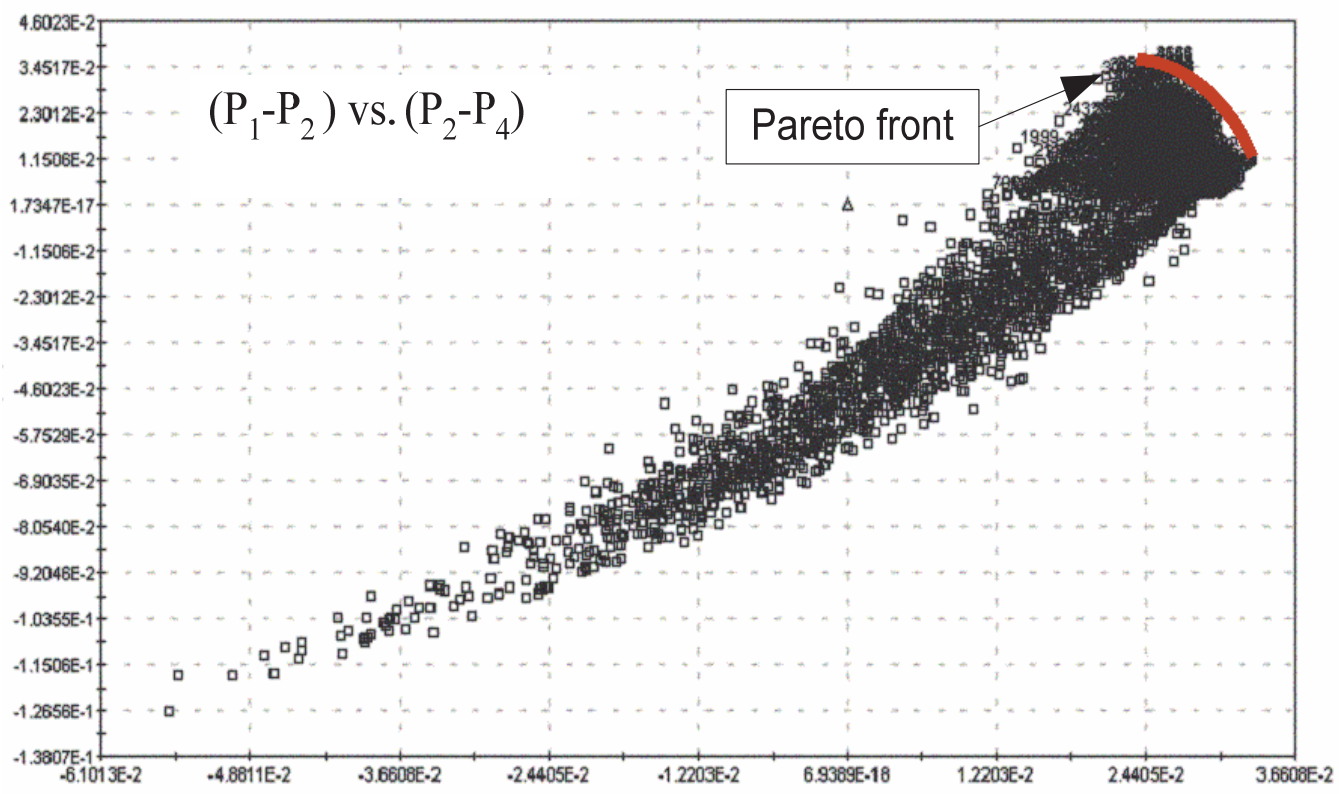

Fig.9 Objectives scatter plot with the Pareto front location indicated The second optimisation starts with the results obtained from the previous one and it focuses on minimising the stress in critical location. This step involved MSC/Patran for automatic mesh 
generation and the setup of the FEM model, MSC/P-Thermal for thermal analysis and MSC/Nastran for the stress analysis.

This optimisation may be formalised as follows:

Find the set of design variables $X$ that minimises:

MPS, pr_1,pr_2

Subject to:

$$
K_{l}<\frac{m_{d}}{m_{d}+m_{p}}<K_{u}
$$

$P_{1}>P_{2}$

$M P S-Y T S<0$

pr_1 - $K_{y} Y T S<0$

pr_2 - K $K_{y} Y T S<0$

As a result of the two optimisation processes a blade root design with an improved pressures distribution was achieved, while the stress in critical areas has been reduced and preserved below the prescribed limits. In Table 4, the comparison between the stresses in critical locations of the original shape and the ones relating to the optimised shape is presented.

Table 4 Blade root optimisation: results.

\begin{tabular}{|c|c|c|c|c|c|}
\hline & Unit & $\begin{array}{l}\text { Stress } \\
\text { Original } \\
\text { Shape }\end{array}$ & $\begin{array}{l}\text { Stress } \\
\text { Final } \\
\text { Shape } \\
\end{array}$ & $\begin{array}{l}\text { Final vs. } \\
\text { Original }\end{array}$ \\
\hline \multirow{4}{*}{ 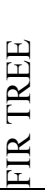 } & area1 & $\mathrm{MPa}$ & 407 & 294 & $-28 \%$ \\
\hline & area2 & $\mathrm{MPa}$ & 416 & 396 & $-5 \%$ \\
\hline & pr_1 & $\mathrm{MPa}$ & 215 & 231 & $7 \%$ \\
\hline & pr_2 & $\mathrm{MPa}$ & 222 & 184 & $-17 \%$ \\
\hline \multirow{8}{*}{ 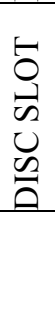 } & area3 & $\mathrm{MPa}$ & 473 & 353 & $-25 \%$ \\
\hline & area4 & $\mathrm{MPa}$ & 717 & 751 & $5 \%$ \\
\hline & area5 & $\mathrm{MPa}$ & 677 & 763 & $13 \%$ \\
\hline & $p r \_1$ & $\mathrm{MPa}$ & 215 & 231 & $7 \%$ \\
\hline & $p r \_2$ & $\mathrm{MPa}$ & 222 & 184 & $-17 \%$ \\
\hline & $P 1-P 2$ & & 0.0529 & 0.0087 & improved \\
\hline & P1-P4 & & 0.0021 & 0.0210 & improved \\
\hline & $P 2-P 4$ & & -0.0610 & 0.0123 & improved \\
\hline
\end{tabular}

Note that the majority of the stresses and contact pressures have been significantly improved. The achieved stress reduction is up to $28 \%$.

The optimised shape presents also a smoother distribution of sectional tensions (cf. rupture criteria), as presented in Fig. 10.
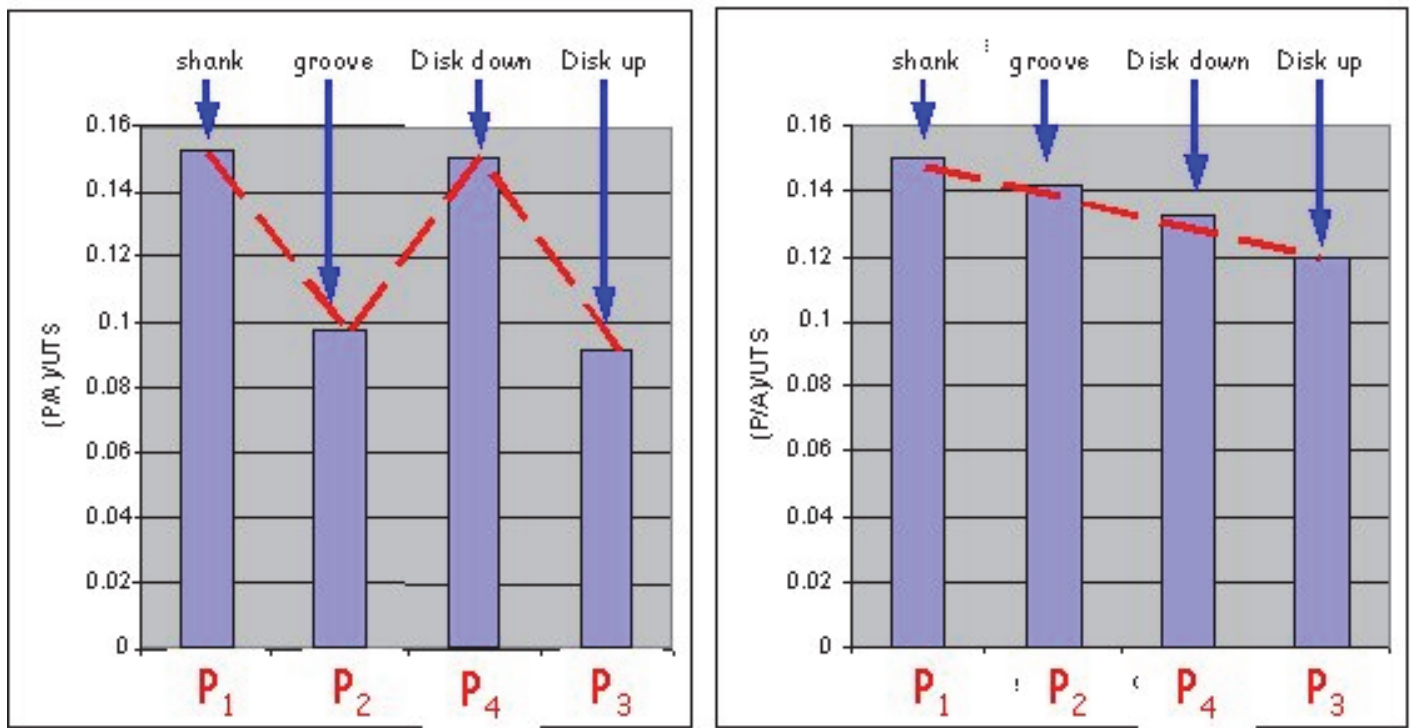
Fig. 10 Distribution of sectional tensions (a) before and (b) after blade root optimisation

\section{Robustness assessment}

It is well known that an optimal design can be very sensitive to small changes in the design parameters, as well as those in the operational environment (Marczyk 2000). The uncertainty in the input parameters results in the variability in the output performance parameters that may lead to performance degradation or even to failure when certain failure-related constraints are violated. Therefore, it is often more sensible to settle for solutions that are only "sufficiently good" but robust in the presence of such variations. Robust design was pioneered by Taguchi (Taguchi 1987) in order to improve engineering productivity and the quality of manufactured goods. The objective of a robust design is generally two-fold: firstly, to achieve the mean response values as close as possible to the prescribed target and, secondly, to reduce the variability in the performance parameters under the known variability of the input parameters (Koch et al 2004). Among the available techniques for the assessment of the robustness, which include Design of Experiments and sensitivity based estimation using first and second order Taylor's expansion; Monte Carlo Simulation is widely regarded as the most appropriate method for analysing responses of systems to uncertain inputs. Robustness can be quantified by expressing the difference between the mean and the limit values in terms of the number of standard deviations. This number is often referred to as sigma-level.

Monte Carlo simulation was used to assess the quality of the blade root design in relation to failure-related constraints used in optimisation. These are:

$$
\begin{aligned}
& P_{1}-P_{2}>0 \\
& M P S-Y T S<0 \\
& p r \_1-K_{y} Y T S<0 \\
& p r_{-} 2-K_{y} Y T S<0
\end{aligned}
$$

Geometric parameters (the solution of the preceding optimisation) were perturbed with the normally distributed noise characterised with standard deviation of $3 \%$. In order to reduce the required number of simulations without sacrificing the quality of the statistical description of the system behaviour, descriptive sampling was used to generate a population of 500 samples (Saliby 1990).

Table 5 provides the results of the robustness assessment expressed as a sigma-level. It can be seen that while the optimised solution achieves a high sigma-level regarding maximal principal stress and contact pressures, the sigma-level for the constraint $P_{1}-P_{2}$ is unacceptably low at 0.6 .

Optimising for six sigma

To improve the robustness of the blade root design, probabilistic design optimisation formulation, as presented by Koch (Koch et al 2004), was implemented. It combines approaches from structural reliability and robust design with the concepts and philosophy of Six Sigma. Variability is incorporated within all the elements of this probabilistic formulation - input design variable bound formulation, output constraint formulation and robust objective formulation.

The implementation involved an automatic optimisation loop, in which Monte Carlo simulations are performed within each iteration. The overall objective was to determine a blade root design according to the stated criteria, while achieving six-sigma level of design robustness in relation to the prescribed output constraints.

Table 5 Blade root analysis: performance quality results derived from the Monte-Carlo analysis

\begin{tabular}{|l|l|l|l|}
\hline & Mean & StDev & Sigma level \\
\hline$M P S \_1-Y T S$ & -389 & 11.4 & $>10$ \\
\hline$M P S \_2-Y T S$ & -474 & 4.89 & $>10$ \\
\hline$p r \_1-0.6 Y T S$ & -282 & 5.58 & $>10$ \\
\hline$p r \_2-0.6 Y T S$ & 244 & 8.42 & $>10$ \\
\hline$P 1-P 2$ & $5.5 \mathrm{E}-3$ & $8.3 \mathrm{E}-3$ & 0.66 \\
\hline
\end{tabular}

The blade root six-sigma based probabilistic design optimisation formulation is given as follows: Find the set of design variables $X$ that minimises:

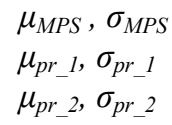


Subject to:

$$
\begin{aligned}
& \mu_{P 1}-6 \sigma_{P 1}>\mu_{P 2}+6 \sigma_{P 2} \\
& \mu_{M P S}+6 \sigma_{M P S}-Y T S<0 \\
& \mu_{p r_{-} 1}+6 \sigma_{p r_{-} 1}-Y T S<0 \\
& \mu_{p r_{-} 2}+6 \sigma_{p r_{-} 2}-Y T S<0
\end{aligned}
$$

The minimisation function has thus been expanded to include minimisation of both the mean and the standard deviation of stress. Also, the output constraints have been reformulated so that the mean plus six standard deviations is within the constraints bounds for all the outputs.

This approach was implemented within modeFrontier design environment and the optimisation was carried out again using a multi-objective genetic algorithm. At each step, 50 Monte Carlo simulations were conducted and the response mean and standard deviation were computed. The overall process involved 1000 optimisation steps and the total computing time was about 5 days. It has been suggested (Marczyk 2000) that one way to improve the overall computational time would be to use the method of stochastic multidisciplinary improvement. In this approach, a set of $N$ random samples is generated around the nominal design. A target location in the performance space is defined and the Euclidean distance of each sample to the target is computed. The best one is chosen as a starting point for the next step of $N$ points. This approach has many aspects in common with the presented robust design approach and it is the subject of our future research. The results from the Six Sigma based probabilistic optimisation is shown in Table 6 in which the new mean and standard deviation values of the output performances are reported. A high sigma level was achieved for all the outputs. While these results may be considered to be overly conservative, the main purpose of the exercise was to demonstrate the overall performance capability.

Table 6 Blade root analysis: performance after the six-sigma based probabilistic optimisation

\begin{tabular}{|l|l|l|l|}
\hline & Mean & StDev & Sigma level \\
\hline MPS_1-YTS & -449 & 7.81 & $>10$ \\
\hline$M P S \_2-Y T S$ & -467 & 3.08 & $>10$ \\
\hline$p r \_1-0.6 Y T S$ & -243 & 4.45 & $>10$ \\
\hline$p r \_2-0.6 Y T S$ & -246 & 5.68 & $>10$ \\
\hline$P_{1}-P_{2}$ & $6.6 \mathrm{E}-3$ & $4.8 \mathrm{E}-4$ & $>10$ \\
\hline
\end{tabular}

\section{Conclusions}

The work presented in this paper was conducted as an attempt to realise Robust Design and Multi Disciplinary Optimisation methodologies in the context of the requirements posed by the aerospace industry, where the overall objectives involve continual reduction of development costs and lead times, while improving the product performance and reliability. In view of the complexity of the product and the need to integrate efforts by teams specialising in various interdependent disciplines, CAD was adopted as the principal repository for product data definition and the principal source of data for various design optimisation processes.

Design optimisation methods require CAD tools to be invoked in an automated loop, in spite of such tools being intended primarily for interactive use. The issues related to variational modelling using parametric CAD models, often leading to generation of incorrect or infeasible geometry, are well documented in the literature. As the permissible range of parameter variation is in practice difficult to predict, the solution was found to be two-fold. First, only a subset of the geometric parameters was selected for optimisation, leading to significantly larger range of permissible variation than when using all parameters in the model. The choice of parameters necessitates detailed knowledge of the problem in hand and judgement by experienced designers, Second, for the chosen set of optimisation parameters, the permissible variation ranges can be adequately estimated using Monte Carlo simulation. As a result, the ability to perform structural optimisations involving both small and large changes in part shape was demonstrated with high probability of producing feasible and satisfactory solutions.

The methodology was implemented and applied in the specific case of gas turbine high pressure disc design. The prescribed design procedure and complexity were considered to be representative for this class of engineering product. The results demonstrated the validity of the overall approach, while the final design was shown to meet relevant design requirements and to achieve significant performance improvements. 


\section{Acknowledgment}

The work presented is part of the EU framework 6 VIVACE project. The authors acknowledge the collaboration from our industrial partners Avio, Rolls-Royce and MTU.

\section{References}

Delfour M.C., J.P. Zolésio, (2001) Shapes and Geometries: Analysis, Differential Calculus, and Optimization. SIAM Philadelphia

Giassi A., Bennis F., Maisonneuve J.J., (2004) Multidisciplinary design optimisation and robust design approaches applied to concurrent design. Structural and Multidisciplinary Optimization 28 (5): 356-371.

Haslinger J., Mäkinen R.A.E. (2003) Introduction to Shape Optimization, and Computation. SIAM, Philadelphia

Hoffman C. M., Joan-Arinyo R. (1998), CAD and the product master model. Computer-Aided Design, Vol. 30, No. 11, pp. 905-918.

Kalsi M., Hacker K., Lewis K., (2001), A Comprehensive Robust Design Approach for Decision Trade-Offs in Complex Systems Design. Journal of Mechanical Design, Vol. 123, No. 1, pp. 1-10. Koch P.N., Yang RJ, Gu L, (2004) Design for six sigma through robust optimization. Structural and Multidisciplinary Optimization, 26 (3-4): 235-248.

Marczyk J, (2000) Stochastic Multidisciplinary Improvement: Beyond Optimization. Proceedings of 8th AIAA/USAF/NASA/ ISSMO Symposium on Multidisciplinary Analysis and Optimization, Long Beach, USA.

Saliby E. (1990) Descriptive Sampling: A Better Approach to Monte Carlo Simulation. J. Opl. Res. Soc. 41(12), 1133-1142.

Shapiro V., Vossler D. (1995) What is a parametric family of solids?, Published in the Proceedings of the Third ACM/IEEE Symposium on Solid Modeling and Applications, Salt Lake City, Utah.

Sang Hun Lee, (2005) A CAD-CAE integration approach using feature-based multi-resolution and multi-abstraction modelling techniques. Computer-Aided Design 37 pp. 941-955.

Bennett J., Fenyes P., Haering W., Neal M. (1998) Issues in Industrial Multidisciplinary Optimization, 7th AIAA/ USAF/NASA/ISSMO Symposium on Multidisciplinary Analysis and Optimization, AIAA 1998-4727, St. Louis,.

Jamshid A. Samareh, (2004)Aerodynamic Shape Optimization Based on Free-form Deformation, 10th AIAA/ISSMO, Multidisciplinary Analysis and Optimization Conference, AIAA-2004-4630, Albany, New York

Melvin R. G., Huffman W. P., Young D. P., Johnson F. T., Hilmes C. L., Bieterman M. B., (1999) Recent Progress in Aerodynamic Design Optimization, International Journal for Numerical Methods in Fluids, Vol. 30, , pp. 205-216.

Raghothama S., Shapiro V., (2002) Topological Framework for Part Families, ASME Transactions, Journal of Computing and Information Science in Engineering, Vol. 2, pp. 246-255. Hoffmann C.M., Joan-Arinyo R. (2002) Parametric modeling. In G. Farin, J. Hoschek, and M.S. Kim, editors, Handbook of Computer Aided Geometric Design, chapter 21, pages 519-541.

Elsevier, Amsterdam

Fenyes P. A., Donndelinger J., Bourassa J., (2002) A New System for Multidisciplinary Analysis and Optimization of Vehicle Architectures, 9th AIAA/ISSMO Symposium on Multidisciplinary Analysis and Optimization, AIAA 2002-5509, Atlanta, Georgia

Carty A., Davies C, (2004) Fusion of Aircraft Synthesis and Computer Aided Design, 10th AIAA/ISSMO Multidisciplinary Analysis and Optimization Conference, AIAA-2004-4433, Albany, New York,

Nemec M., Aftosmis M., Pulliam T., (2004) CAD-Based Aerodynamic Design of Complex Configurations Using a Cartesian Method, 42nd AIAA Aerospace Sciences Meeting and Exhibit, AIAA-2004-113, Reno, Nevada

Baker M. L., Alston K Y., Munson M. J., (2002) Integrated Hypersonic Aeromechanics Tool (IHAT)", 9th AIAA/ISSMO Symposium on Multidisciplinary Analysis and Optimization, AIAA 2002-5512, Atlanta, Georgia

Röhl P.J., He B, Finnigan P.M., (1998) A collaborative optimization environment for turbine engine development, 7th AIAA/USAF/NASA/ISSMO Symposium on Multidisciplinary Analysis and Optimization, AIAA 1998-4734, St. Louis

Giesing J.P., Barthelemy J.F.M. (2007) A summary of industry MDO applications and needs, AIAA 
Kesseler E., van Houten M.H. (2007) Multidisciplinary optimisation of a turbine disc in a virtual engine environment, 2nd European Conference for Aerospace Sciences

EUCASS, Brussel

LeDoux S. T., Herling W. W., Fatta J., Ratcliff R. R., (2004) MDOPT - Multidisciplinary Design Optimization System Using Higher Order Analysis Codes, 10th AIAA/ISSMO Multidisciplinary Analysis and Optimization Conference, Albany, New York

Taguchi G., Chowdhury S., Yuin W. (2004) Taguchi's Quality Engineering Handbook New York: John Wiley \& Sons.

Taguchi, Genichi, "System of Experimental Design” Edited by Don Clausing. New York: UNIPUB/Krass International Publications, Volume 1 \& 2, 1987.

Zhang, Chun; Wang, Hsu-Pin (1998) Robust design of assembly and machining tolerance allocations. IIE Transactions Volume 30, Volume 30, Number 1 pp. 17-29 pp. 17-29

Lee KY, Armstrong CG, Price MA, Lamont JH. (2005) A small feature suppression/unsuppression system for preparing B-Rep models for analysis. In: Proceedings of the 2005 ACM symposium on solid and physical modeling.

Fig. 1 Gas turbine disc

Fig. 2 Typical MDO/RD process flow

Fig. 3 Model Structure

Fig. 4: Parameterized disc

Fig. 5: Parameterised blade root and slot

Fig. 6: Example of a non-feasible geometry

Fig. 7: Parameterisation for preliminary disc design

Fig. 8 Blade root and disc slot design

Fig.9 Objectives scatter plot with the Pareto front location indicated

Fig. 10 Distribution of sectional tensions (a) before and (b) after blade root optimisation 